\title{
Technik der Präparation und Darstellung von Sedimentkernen mit grossem Querschnitt
}

\author{
Von H. Ambühl \\ EAWAG Dübendorf \\ Manuskript eingegangen am 11. November 1985
}

\section{ABSTRACT}

A technique for the preparation and presentation of sediment core samples with large cross section

A boring instrument for the removal of undisturbed samples from lake sediments at any depth is described. The bore tube cuts a sediment core (diameter $110 \mathrm{~mm}$ ) in which the layering structure of a $300 \mathrm{~mm}$ long section can be examined in its natural undisturbed form. The core is sectioned using a knife made out of mineral glass (thin window glass). The core can be fixed with a piston and laid down horizontally. After removing the glass knife, it can be further processed, sampled, etc. Examples of such cores from the Greifensee, the Hallwilersee and from Lake Lucerne (Bay of Gersau) show the amazing detail and individuality of the sediments.

Mit dem «Bohrlot für Grossproben» [1] ist es möglich, aus dem Sediment von Seen Bohrkerne von $110 \mathrm{~mm}$ Durchmesser in völlig ungestörter Schichtlagerung zu gewinnen. Die weitere Verarbeitung erfordert, dass der Kern geöffnet wird, um die Schichtstruktur sichtbar zu machen.

Bei dieser Präparation handelt es sich im Prinzip um die von H. Züllig im Jahre 1953 eingeführte Technik, bei der der Kern mit einer durchsichtigen Lamelle längs aufgeschnitten wird [2]. Die eine Hälfte wird verworfen, während die hinter der Lamelle verbleibende Kernhälfte den Aufschluss (bzw. das Schnittbild) liefert. Diese Methode lässt sich an Kernen mit geringem Durchmesser problemlos praktizieren. Um das Sediment (namentlich für didaktische und auch dokumentarische Zwecke) breitflächiger, «wirklicher» sichtbar machen zu können, haben wir das bewährte Zülligsche Verfahren auf Grossproben adaptiert. Die Methode wird seit 1982 angewendet.

\section{Das Gerät}

Es besteht aus dem 1975 beschriebenen Bohrgerät [1] und neu entwickelten Bohrrohren (Abb. 1, 2). Diese Rohre besitzen diametral angeordnete Längsschlitze zur Aufnahme eines gläsernen Messers sowie (vor den Schlitzen plaziert) ein $300 \mathrm{~mm}$ hohes Fenster. Die zwischen Schlitzen und Fenster verbleibenden Stege sind durch rostfreie Metallprofile versteift. Am oberen und unteren Ende der Schlitze befinden sich Bohrungen zur Auf- 


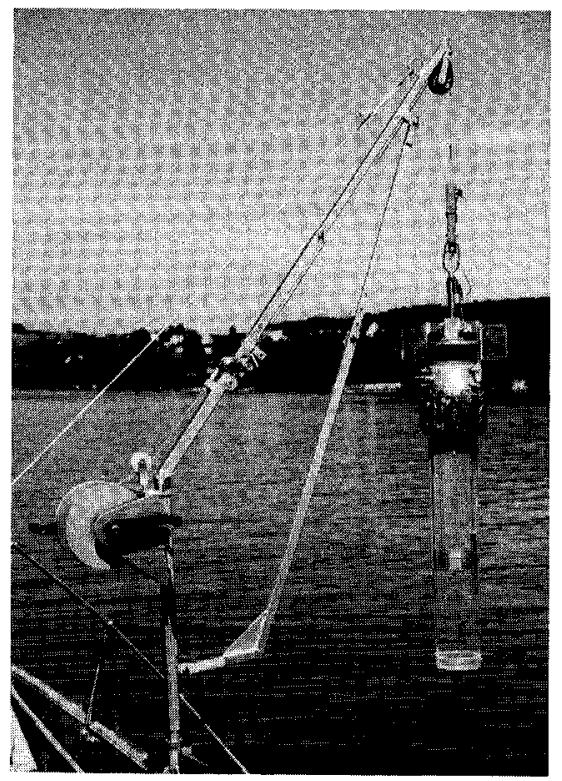

Abb. 1. Bohrgerät mit dem neuen Stechrohr. Fenster und Schlitze sind verklebt. Figure 1. Boring instrument with the new cutting tube. Window and slits are closed by tape.

nahme von Führungsprofilen für das Messer (Abb.2). Während H. Züllig zum Aufschneiden des Bohrkerns dünnes Plexiglas verwendet, ist dies bei unserem Rohr-Innendurchmesser von $110 \mathrm{~mm}$ nicht möglich infolge der mangelnden Stabilität des Plexi-Materials. Wir verwenden deshalb Mineralglas (das dünnste erhältliche Fensterglas, $1 \mathrm{~mm}$ stark); einzig dieses Material ist steif genug und auch so hart, dass man eine Messerschneide anschleifen kann. Um nun dafür zu sorgen, dass das Messer beim Schneidvorgang auf Anhieb den gegenüberliegenden Schlitz findet (ein mehrfacher Versuch würde das Bild sofort zerstören), müssen die erwähnten Führungsprofile verwendet werden. Gelingt das Durchstecken der Führungen nicht aufs erste Mal, so stört dies das Sedimentprofil nicht. Der Schnitt mit dem Glasmesser gelingt nach unserer Erfahrung sodann fast ausnahmslos auf Anhieb. Das Fenster ist mit einem satt passenden Rohrstück exakt ausgefüllt. Exakt deshalb, weil das Rohr durch den Fenstereinschnitt stark geschwächt ist, beim Einstechen ins Sediment aber grössere mechanische Kräfte auftreten. Durch das genau eingepasste Fensterstück wird der Druck weitergeleitet, und das Rohr kann sich nicht verformen oder brechen. (Die Glasmesser werden von einer Zürcher Spezialfirma angefertigt). Vor der Probenahme werden sämtliche Schlitze, Öffnungen usw. mit wasserfestem Verpackungs-Klebeband verklebt und das Gerät auf Dichtigkeit geprüft. Es ist hier grundsätzlich zu bemerken, dass man auch bei einem derart wässrigen, puddingartigen Material wie dem geschichteten Seesediment keine sauberen Schnitte erwarten darf, wenn man nicht scharfe Messer benützt. Das gilt genauso für die Stechkante am unteren Rohrende wie auch für das Messer zum Zerteilen des Kerns. 


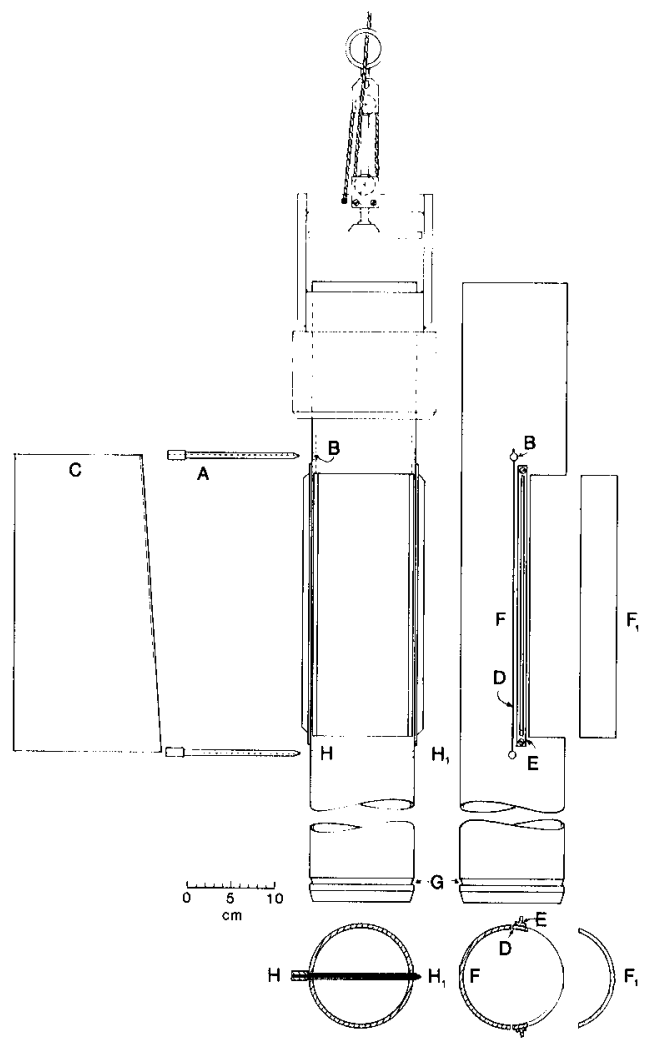

Abb. 2. Schemaskizze des Bohrrohres. Mitte: Ansicht von vorne, rechts: Seitenansicht. Die beiden Führungsprofile (A) werden in die Bohrungen B gesteckt (siehe Schnittbild $\mathbf{H}-\mathbf{H}_{1}$ ). Das Messer (C) wird sodann, durch die Nuten der Führungen geleitet, durch die Schlitze D hindurchgeschoben. Die Schlitze sind durch Metallprofile (E) stabilisiert. In der Ansicht (Mitte) und im Querschnitt F-F $F_{1}$ ist das Fenster F weggelassen; es ist rechts aussen separat dargestellt. Die Ringnut $\mathrm{G}$ am unteren Rohrende dient zur Befestigung des Verschlussdeckels und (eventuell) als Auslösersignal und Wegbegrenzung für einen mobilen Verschlussmechanismus [1]. Der Gerätekopf ist nach [1] schematisch angedeutet.

Figure 2. Schematic diagram of the bore tube. Middle: plane view. Right: side view. The two guide pegs (A) are inserted in the bore holes $\mathrm{B}$ (see cross section $\mathrm{H}-\mathrm{H}_{1}$ ). The knife $(\mathrm{C})$ can then be slid along the groove in the pegs, via the slits (D) through the tube. The slits are strengthened by metal reinforcement $(\mathrm{E})$. In the plan view and in the cross section $F-F_{1}$ the window $(F)$ has been omitted. It is shown separately on the far right. The ring groove (G) at the lower end of the tube serves to secure the fastening lid and (possibly) as a signal and obstruction for an automatic closing mechanism [1]. The top of the instrument is shown diagrammatically according to [1].

\section{Die Technik der Präparation}

Das Bohrgerät wird nach der Probenahme möglichst mit einem Motorkran hochgehievt und sehr sorgfältig über die Wasseroberfläche gehoben. Die obersten 5 bis $7 \mathrm{~cm}$ des Bohrkerns sind äusserst inkonsistent und werden schon durch schwache Dreh- und Neigungsbewegungen durcheinander gemischt, was die Probe für weitere Verwendung unbrauchbar macht. Das Bohrgerät muss deshalb äusserst gleichmässig und ruhig bewegt 
werden. Drehbewegungen, welche infolge des Dralls des Kranseils stets drohen, müssen sofort gestoppt oder von Anfang an vermieden werden, ebenso jede Auslenkung aus der Vertikallage. Diese Bedingung kann nur eingehalten werden, wenn ein Kran mit genügender Höhe zur Verfügung steht, um das Bohrgerät frei hängend über die Bordkante zu bringen (Abb. 1). Wenn das Gerät aus dem Wasser auftaucht, wird das untere Rohrende mit einem Deckel, der später noch zu fixieren ist, provisorisch verschlossen, mit dem Kran ganz hochgezogen und mitsamt dem Deckel auf einen vorbereiteten Platz abgestellt. Ist der Kern zu lang, lässt man unten eine entsprechende Länge herausfliessen, ist er zu kurz, so kann er durch Eindrücken von Distanzstücken gehoben werden, damit das Fenster vollständig ausgenützt ist. Der Deckel wird jetzt mit einer vorher justierten Bandage befestigt. Der schwere Gerätekopf wird abgenommen und das Bohrrohr mit Kern in ein bereitstehendes Laborstativ eingespannt (Abb. 3).

Als nächstes muss das überstehende Wasser abdrainiert werden. Zu diesem Zweck werden die Verklebungen auf den Schlitzen bis knapp oberhalb der Sedimentoberfläche eingeschnitten und das Wasser schliesslich soweit abdrainiert, dass das Festmaterial nicht
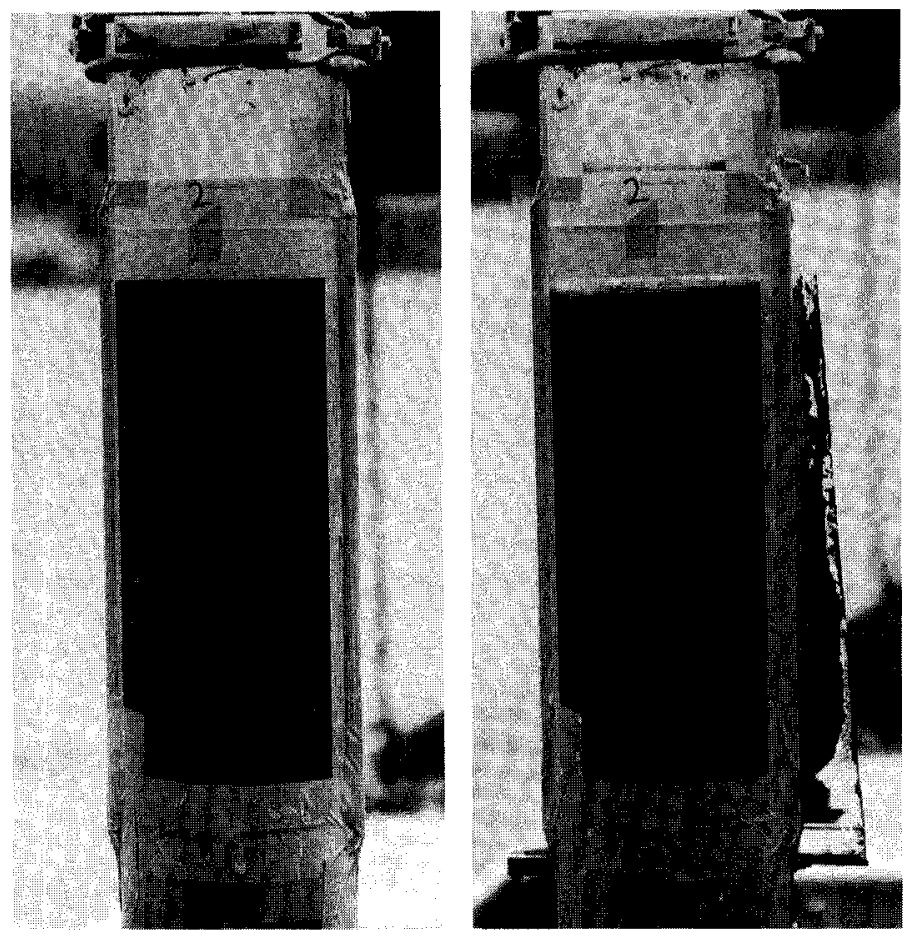

Abb. 3. Bohrrohr mit einem frisch gestochenen Sedimentkern.

Figure 3. Bore tube with a freshly dug out sediment core.

Abb. 4. Mit Hilfe der eingesetzten Führungsstäbe wurde das Glasmesser durch den Kern hindurchgestossen und kommt auf der Gegenseite zum Vorschein. Die Verklebungen und das Fenster werden jetzt entfernt.

Figure 4. The glass knife has been stuck through the core by means of the guide pegs and appears at the other side. The clips and the window are now removed. 
mehr fliessen (und sich dadurch mischen) kann. Jetzt werden die beiden Führungsprofile eingesetzt, die dazwischen liegenden Schlitze auf volle Länge aufgeschnitten, sodann das Glasmesser in die Nuten der Führungen eingesetzt und durchgestossen. Ober- und Unterkante des «Messers» laufen parallel; sie müssen genau in die Führungsnuten passen. Vorder- und Hinterkante sind dagegen nicht parallel, sondern die geschärfte Vorderkante ist schräg angeordnet, damit die Schneide den Schlitz nicht auf der ganzen Länge gleichzeitig durchdringen muss. Nachdem nun das Messer auf der Gegenseite zutage getreten und vollständig durchgesteckt ist (Abb.4), werden die Verklebungen rings um das Fenster aufgeschnitten und der Fenstereinsatz herausgenommen. Der vor dem Glasmesser liegende Teil des Kerns wird vorsichtig entfernt. Die Scheibe wird mit Schwamm und Wasser gesäubert und schliesslich mit einer Spritzflasche nachgereinigt. Hinter der Scheibe steht nun das (hoffentlich - es gelingt nicht immer ...) ungestörte Sedimentprofil - in den meisten Fällen ein faszinierender Anblick (Abb. 7).

Wie rasch diese Präparation zu erfolgen hat, hängt davon ab, wie rasch das Sediment, das jetzt vom hydrostatischen Druck entlastet ist, Gasblasen entwickelt. So muss das methanreiche Material aus dem Greifensee unverzüglich und sehr rasch, d.h. innert Minuten,

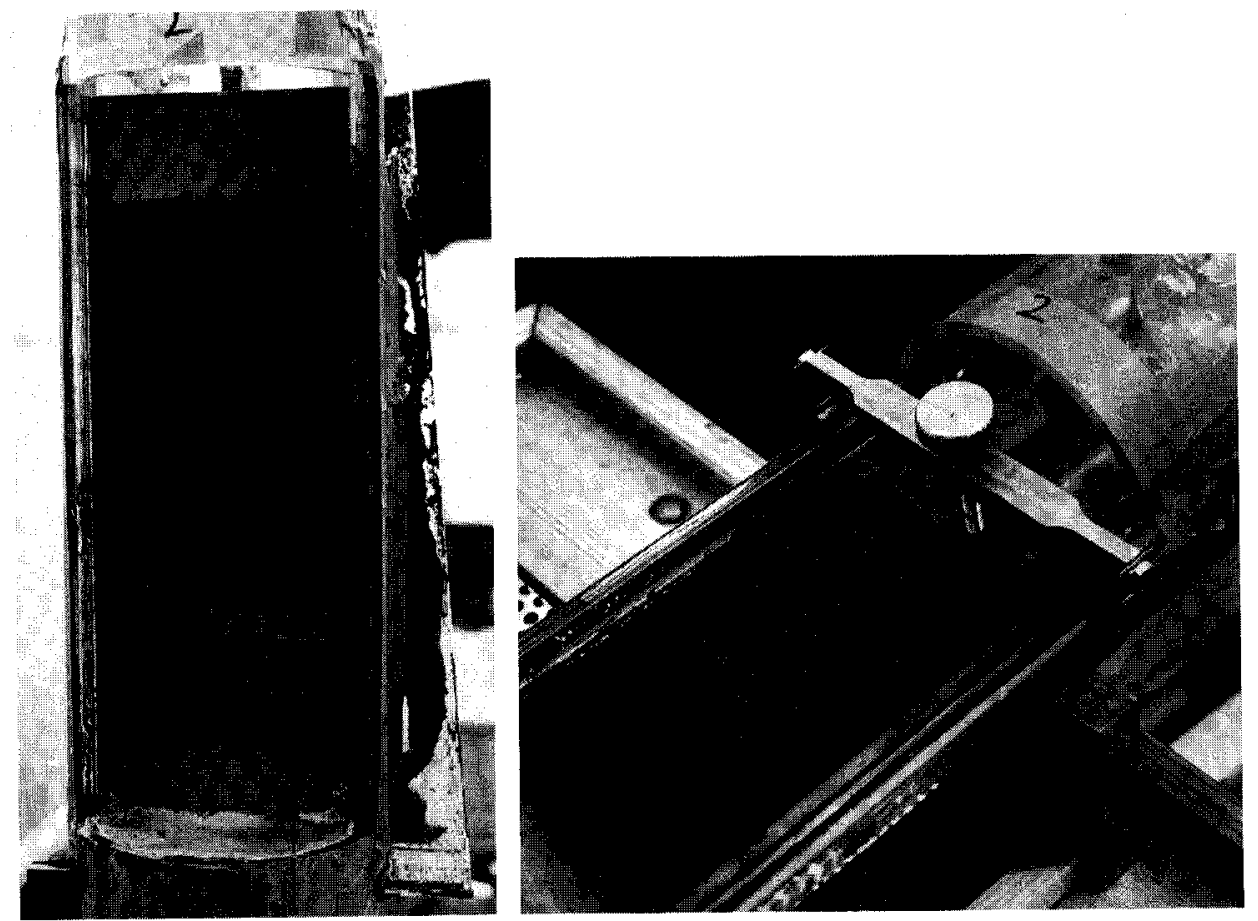

Abb. 5. Der Kern ist mit einem von oben eingeführten und am Rohrrand fixierten Kolben gesichert. Figure 5. The core is fixed and secured from the top and around the sides using a piston.

Abb. 6. Nach Umlegen in die Horizontallage wurde das Glas entfernt und der Kolben mit einer Verschraubung gesichert. Der Kern ist jetzt zu weiterer Bearbeitung (Subsampling, Messungen) und zum Transport bereit. Figure 6. After inverting into the horizontal position the glass is removed and the piston secured with a screw clamp. The core is now ready for further processing (subsampling, measurements, etc.) and for transportation. 
bearbeitet werden, wenn man noch passable Photodokumente erhalten will. Das wesentlich gasärmere Sediment des Vierwaldstättersees stellt trotz der grösseren Wassertiefe geringere Anforderungen; dafür sind die jüngsten Ablagerungen einzelner Seeteile derart inkonsistent, dass sie nur mit grosser Mühe und nach manchen Wiederholungen überhaupt störungsfrei präpariert werden können.

\section{Die Photodokumentation}

Die im Anhang abgebildeten Beispiele sind ausnahmslos «Falschbelichtungen», dies mit der Absicht, die Hell-Dunkel-Struktur besser zur Geltung zu bringen. Von der Überlegung ausgehend, dass schwarze Zonen auch bei Überbelichtung dunkel erscheinen, helle dagegen aufgehellt werden, verwenden wir standardmässig eine gewisse Überbelichtung, welche das Gesamtbild heller erscheinen lässt als das Original und Einzelheiten klarer wiedergibt. Die Aufnahmen werden auf Farbmaterial gemacht, da die hellen Schichten sehr oft eine gut wahrnehmbare Eigenfarbe besitzen. Verwendet wird der Film Kodachrome 64 (Empfindlichkeit 64 ASA). Die Belichtungsautomatik der Kamera und das Automat-Blitzgerät (letzteres auf Nahbereich eingestellt) werden auf 64 und für eine zweite Serie auf 32 ASA eingestellt. Die Blitzbelichtung erfolgt aus $45^{\circ}$ seitlich, in Abständen von etwa 80,100 und $120 \mathrm{~cm}$ von der Kernmitte. Um Reflexe soweit möglich zu vermeiden, muss der Bereich hinter der Kamera mit einem dunklen Schirm abgedeckt werden. Bei Sonnenschein ist auch das Bildfeld $\mathrm{zu}$ beschatten. Um das $30 \mathrm{~cm}$ hohe Fenster formatfüllend aufzunehmen, ist ein Nah-oder Makroobjektiv erforderlich.

Abb. 7. Beispiele von Sedimentaufschlüssen aus verschiedenen Seen.

a) Greifensee, $32 \mathrm{~m}$ tief, Sommer 1985: Feine Stratifikation, in typischer Abfolge der Schichten. Jedes

Hell-Dunkel-Schichtpaar hat ein individuelles Aussehen, jede helle (Winter-Frühjahrs-Schicht) hat eine individuelle, in jedem neuen Kern leicht wieder zu findende (reproduzierbare) Struktur. Beispiele: $\mathrm{A}=$ auffällig helle Doppelschicht von 1977, B = relativ mächtige, fein strukturierte Schicht 1974,

$\mathrm{C}=$ Hochwassereinschwemmung von etwa 1967. Der Aufschluss besitzt ein unverwechselbares, jederzeit leicht erkennbares Aussehen. Der Beginn der dunklen Schichten fällt etwa in die Jahrhundertwende.

b) Hallwilersee, $45 \mathrm{~m}$ tief, Sommer 1985: Besonders schönes Beispiel einer ungestörten Schichtablagerung. Auch hier ist die regelmässige Abfolge der Hell-Dunkel-Schichtpaare bemerkenswert. Besonders zu beachten ist wiederum der individuelle Charakter jeder einzelnen Schicht, die sich in einem derartigen Aufschluss einzeln präparieren bzw. beproben lassen. Der Beginn der dunklen Schichten ist etwa 1910 anzusetzen.

c) Vierwaldstättersee, Becken von Gersau, $214 \mathrm{~m}$ tief, Herbst 1984: Schichtfolge, welche vor allem durch Hochwassereinschwemmungen (helle Zonen) und durch unregelmässig auftretende anaerobe Phasen geprägt ist, letzteres die Folge der bei der grossen Wassertiefe oft ungenügenden Zirkulation bzw. Sauerstoffversorgung.

Figure 7. Example of sediment cores from different lakes.

a) Greifensee, $32 \mathrm{~m}$ deep, summer 1985. Fine stratification, a typical layered structure. Each light-dark layer pair has an individual appeareance. Each light (winter half year) layer has an individual structure which can be found reproducibly in each new core. For example, $\mathrm{A}=$ conspicuously bright double layer from $1977, \mathrm{~B}=$ relatively

obvious fine structured layer from $1974, \mathrm{C}=$ flood wash out from ca. 1967. The information is unmistakable and each time easily deduced. The beginning of the dark layering began around the start of the century.

b) Hallwilersee, $46 \mathrm{~m}$ deep, summer 1985 . Especially good example of an undisturbed layered structure. Also

here, the regularly repeating light-dark sequence is obvious. Worthy of note is once again the individual character of each layer, which allows their detailed examination. The start of the darker layers occurred around 1910.

c) Lake Lucerne (Bay of Gersau), $214 \mathrm{~m}$ deep, autumn 1984. The layering is strongly indicative of being caused by regular flood wash out (light zones) and unregularly occurring anaerobiosis, the latter being typical of deep waters with insufficient turnover with concomitant oxygen depletion. 


\section{Mechanische Sicherung des Kerns}

Nach erfolgter Photodokumentation steht der Kern zu weiterer Verwendung bereit. Um das Ausgasen und vor allem die Ansammlung grosser Gasblasen zu vermeiden, wird er mit einem genau passenden Halbkolben, der an einer Stange aufgeschraubt ist und hinter der Scheibe bis zur Sedimentoberfläche hinuntergestossen wird, fixiert (Abb. 5). Die den Kolben tragende Stange wird am Rohrrand mit einer speziellen Muffe festgeklemmt und das Ganze sodann in Horizontallage umgelegt. Die Scheibe wird sorgfältig weggezogen, der Kolben mit einem unter dem Rohr durchgezogenen Metallband und einem Querjoch fixiert und die Stange ausgeschraubt (Abb.6). Der Kern liegt jetzt offen da und kann weiterbearbeitet werden. In Sedimenten eutropher Seen sind die Jahresschichten so gut sichtbar, dass sie einzeln abgezählt und beprobt werden können. In diesem Zustand ist der Kern erheblich transportfähiger als hinter Glas, weil die sich bildenden Blasen jetzt auf dem kürzesten Weg austreten können.

\section{Zusammenfassung}

Beschreibung eines Bohrgerätes zur Entnahme von ungestörten Proben aus dem Sediment von Seen, dies aus beliebiger Wassertiefe. Das Bohrrohr erlaubt, den Bohrkern (Durchmesser $110 \mathrm{~mm}$ ) auf einer Länge von maximal $300 \mathrm{~mm}$ längs aufzuschneiden und die Schichtungsstruktur im ungestörten Zustand zu dokumentieren. Der Schnitt erfolgt mit einem Messer aus dünnem Mineralglas (Fensterglas). Der Kern kann mit einem Kolben «fixiert» und horizontal umgelegt werden. Nach Entfernung des Glasmessers kann er sodann weiterverarbeitet, beprobt usw. werden. Beispiele derart präparierter Kerne aus dem Greifensee, dem Hallwilersee und dem Vierwaldstättersee (Becken von Gersau) zeigen die erstaunliche Feinheit und Individualität der Sedimente.

\section{VERDANKUNG}

Der Autor dankt seinen Mitarbeitern Dr. P. Bossard, Dr. U. Uehlinger und F. Stössel für wertvolle Kritik und tatkräftige Hilfe bei den Probenahmen sowie Tony Mason für die Übersetzung ins Englische.

Die Geräte wurden von der Werkstatt der Technischen Versuchsstation Tüffenwies der EAWAG hergestellt. Die heikle Bearbeitung der Plexiglasrohre durch die Herren E. Schwager und H. Suter sei besonders hervorgehoben.

\section{LITERATURVERZEICHNIS}

1 Ambühl, H., und Bührer, H.: Zur Technik der Entnahme ungestörter Grossproben von Seesediment: ein verbessertes Bohrlot. Schweiz. Z. Hydrol. 37 (1), 175-188 (1975).

2 Züllig, H.: Ein neues Lot zur Untersuchung der obersten Schlammschichten, zur Messung des Sedimentabsatzes und zur Erfassung bodennaher Wasserschichten. Schweiz. Z. Hydrol. 15 (2), 275-284 (1953).

Adresse des Autors: EAWAG, Überlandstrasse 133, CH-8600 Dübendorf, Switzerland. 